\title{
Structural heterogeneity of diamond-containing detonation material
}

\author{
A. Korets ${ }^{1, a}$, A. Krylov ${ }^{2}$, and E. Mironov ${ }^{3}$ \\ 1 Siberian Federal University, 26 Kirensky str., 660074 Krasnoyarsk, Russia \\ 2 Institute of Physics, SB RAS, 660036 Krasnoyarsk, Russia \\ ${ }^{3}$ Krasnoyarsk Institute of Railway Transport (Filial of Irkutsk State University of Railway Engineering), 660028 Krasnoyarsk, \\ 89 L. Ketskhoveli str., Russia
}

Received: 21 December 2009 / Received in final form: 11 May 2010 / Accepted: 30 June 2010 Published online: 17 September 2010 - (C) EDP Sciences

\begin{abstract}
Diamond-containing material (DCM) synthesized by detonation was separated into fractions. Raman and infrared spectra and X-ray diffraction patterns of the individual fractions were measured. The particles of this material were characterized by the variable ratio of the diamond $\left(s p^{3}\right)$ and non-diamond components. The distribution of $s p^{3}$-grains in the particles was of complicated character. The fine DCM particles contained an insignificant amount of diamond. The influence of density fluctuations on the DCM formation is discussed.
\end{abstract}

\section{Introduction}

Synthesis of materials by means of dynamic (detonation) methods proceeds under non-equilibrium physical and chemical conditions. In the expanding field of rapid synthetic methods, understanding and overcoming structural heterogeneity in the products is a scientific and technical challenge. Structurally inhomogeneous diamondcontaining material (DCM) was originally known as detonation nanodiamond or ultradispersed diamond $[1,2]$. This material comprises fragments of molecules, functional groups, $s p^{2}$ hybridized carbon (the non-diamond part) and $s p^{3}$ (diamond) grains. Different researchers have come to disparate conclusions about the structure, stability, and a formation time of these components. Structural heterogeneity of DCM is evidenced by a scatter in the measurements of material characteristics and is believed to be the result of non-equilibrium conditions. In DCM, light fractions obtained by milling and centrifugation are observed with distinct characteristics $[3,4]$. These results may reflect a complicated distribution of the diamond areas in the particles.

The aim of this work is to better understand the diamond grain distribution and elucidate the composition of DCM admixtures (the non-diamond part) and the fractions using molecular spectroscopy techniques accounting for the non-equilibrium influence on the synthesis.

\section{Materials and methods}

Raman spectra of backward scattering were recorded in the frequency range $100-4000 \mathrm{~cm}^{-1}$ with a $1 \mathrm{~cm}^{-1}$ reso-

\footnotetext{
a e-mail: korets1947@rambler.ru
}

lution Bruker RFS 100/S Fourier spectrometer. A Coherent Compass 1064-1500N Nd:YAG laser (800 mW) with diode pumping was used. A T64000 Jobin Yvon spectrometer was used to measure spectra in the region 1000$2000 \mathrm{~cm}^{-1}$. Argon ion laser radiation at $514.5 \mathrm{~nm}$ with $37 \mathrm{~mW}$ directed onto the sample was used for excitation. Measurements were taken through a microscope in a $180^{\circ}$ geometry.

IR spectra were obtained in the $400-4000 \mathrm{~cm}^{-1}$ region using a Vertex-44 Fourier spectrometer and a Specord M82 unit with computer data processing. The DCM powder was mixed with $\mathrm{KBr}$ and pressed into a pellet.

The DCM synthesis took place in a preservation atmosphere of either carbon dioxide (DCM-1) or molecular nitrogen (DCM-2). The DCM-1 and DCM-2 samples were synthesized from a trinitrotoluene/hexogen (TNT/RDX) mixture and extracted from the detonation soot by thermoxidation in the presence of boron anhydride and chloric acid oxidation, respectively.

The DCM-2-1 and DCM-2-2 samples were obtained in the same explosion chamber, with a volume of $0.175 \mathrm{~m}^{3}$ and molecular nitrogen at an initial pressure of $0.7 \mathrm{MPa}$. The samples were isolated using the same method (chloric acid oxidation). High explosives were a mixture of trinitrobenzol/hexogen (DCM-2-1), and trinitroresorcin/hexogen or picric acid/hexogen (DCM-2-2).

We also studied the detonation soot isolated from metallic admixtures (a technological mark of this sample is UDDG) by hydrochloric acid treatment.

Fraction separation was carried out in a ZLN-2 centrifuge at a speed of up to $7000 \mathrm{rpm}$. The DCM concentration in hydrosol was $10-50 \mathrm{~g} / \mathrm{l}$. Centrifugation time was 5 min. Table 1 shows the centrifugation results. 
The European Physical Journal Applied Physics

Table 1. The results of DCM centrifugation. TNB is trinitrobenzol, RDX is hexogen, and TNR is trinitroresorcin. The mass of the light fraction was obtained by centrifugation with 3000, and $7000 \mathrm{rpm}$. HE refers to the high explosive precursor mixture.

\begin{tabular}{|c|c|c|c|c|c|}
\hline Samples & $\begin{array}{c}\text { Composition } \\
\text { of original } \\
\mathrm{HE}\end{array}$ & $\begin{array}{c}\text { Light fraction } \\
\text { mass / mass } \\
\text { of the sample, } \\
\text { wt. } \%\end{array}$ & Samples & $\begin{array}{c}\text { Composition } \\
\text { of original } \\
\mathrm{HE}\end{array}$ & $\begin{array}{c}\text { Light fraction } \\
\text { mass/mass } \\
\text { of the sample, } \\
\text { wt. } \%\end{array}$ \\
\hline $\begin{array}{l}\text { DCM-2-1 } \\
3000 \text { rpm }\end{array}$ & $\begin{array}{c}\mathrm{TNB} / \mathrm{RDX} \\
60 / 40\end{array}$ & 3.50 & $\begin{array}{c}\text { DCM-1 } \\
7000 \text { rpm }\end{array}$ & $\begin{array}{c}\text { TNT/RDX } \\
\text { Technological } \\
\text { sample } \\
\text { (Krasnoyarsk) }\end{array}$ & 0.05 \\
\hline $\begin{array}{l}\text { DCM-2-2 } \\
3000 \mathrm{rpm}\end{array}$ & $\begin{array}{c}\text { TNR/RDX } \\
70 / 30\end{array}$ & 2.20 & $\begin{array}{c}\text { DCM-1 } \\
3000 \mathrm{rpm}\end{array}$ & $\begin{array}{c}\text { TNT/RDX } \\
\text { Technological } \\
\text { sample } \\
\text { (Krasnoyarsk) }\end{array}$ & 2.10 \\
\hline
\end{tabular}

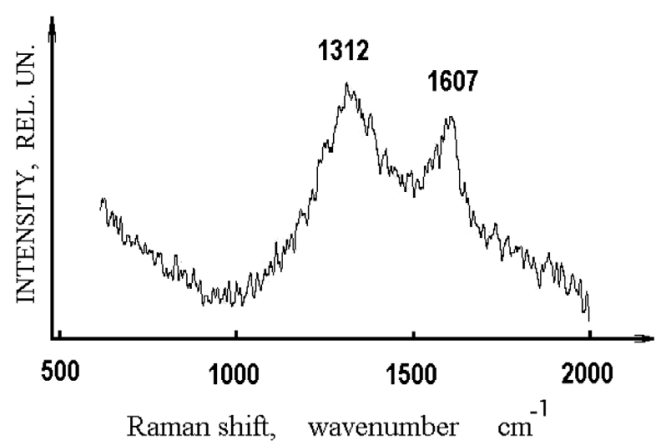

Fig. 1. Raman spectrum of UDDG (refined detonation soot). This sample was treated to remove metallic admixtures.

X-ray diffraction (XRD) patterns for the DCM samples were obtained using an XRD-6000 Shimadzu diffractometer.

\section{Results}

Figure 1 shows the Raman spectrum of a UDDG sample (detonation soot). The broad band at $1312 \mathrm{~cm}^{-1}$ is likely to be a superposition of several contributions [5-7]; one of them is the phonon mode of $\mathrm{F}_{2 g}$ symmetry $\left(1333 \mathrm{~cm}^{-1}\right.$ for bulk diamond). It is reasonable to suggest that the $1607 \mathrm{~cm}^{-1}$ band is due to the large amount of $s p^{2}$ carbon ( $\mathrm{G}$ band) (Fig. 1). The position of the G-band maximum is normally dependent on the configuration of $s p^{2}$ carbon. The molecular groups likely have an effect on the position of the maximum of both bands.

The Raman and IR spectra of the samples (DCM-1 and DCM-2) are showed in Figures 2-7. It is unlikely that the non-diamond $\left(s p^{2}\right)$ carbon is exclusively responsible for these features of the Raman spectra. Additional molecular groups and fragments can also be formed in DCM particles under rapid quenching followed by condensation, as shown in previously published IR spectra of DCM [8-11]. According to [5], the broad asymmetric Raman band between 1500 and $1700 \mathrm{~cm}^{-1}$ originates from $\mathrm{O}-\mathrm{H}$ bending vibrations and $\mathrm{C}=\mathrm{O}$ stretching vibrations. Molecular

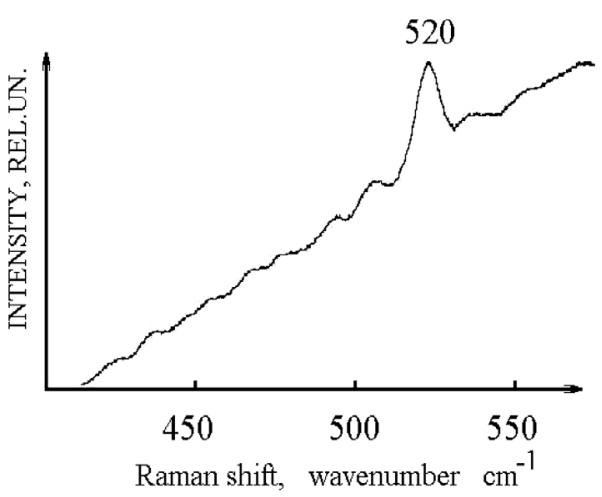

Fig. 2. Raman spectrum of DCM-1 in the range 400$650 \mathrm{~cm}^{-1}$. A similar band appears in the IR spectra of DCM as well. Comparison of IR and Raman spectra of DCM-1 reveals some common spectral features (500-600 $\mathrm{cm}^{-1}, 1200-1330$, 1600-1650, and $3200-3550 \mathrm{~cm}^{-1}$ ), because the DCM particles are structurally nonuniform [8].

groups and fragments of $\mathrm{R}-\mathrm{X}-\mathrm{N}=\mathrm{O}(\mathrm{X}=\mathrm{O}, \mathrm{C}, \mathrm{N})$ and $\mathrm{X}-$ $\mathrm{NO}_{2}(\mathrm{X}=\mathrm{O}, \mathrm{N})$ may be found [8] and will give characteristic bands in the DCM Raman spectra. Vibrations of these groups may overlap with and obscure the diamond and graphite bands. The $520 \mathrm{~cm}^{-1}$ band is related to bending vibration in these groups. Ester groups, which constitute structural units of heterogeneous DCM particles, also complicate the spectra. Their concentration depends on the type of the DCM sample [8-11].

A real technological product (i.e. a product synthesized in large quantities) contains carbon structures with a complex morphology. Such structures are also formed under laboratory conditions when the synthesis conditions are not optimal [12]. These carbon structures are expected to affect the Raman spectrum of DCM, possibly through Dor G- spectral bands.

Figures 3 and 4 show IR spectra of the centrifugation fractions. IR spectra of the DCM-1 light and heavy fractions were largely the same.

XRD intensity of the diamond band for the light fraction was low, as shown in Figure 8. XRD intensity for the 
A. Korets et al.: Structural heterogeneity of diamond-containing detonation material

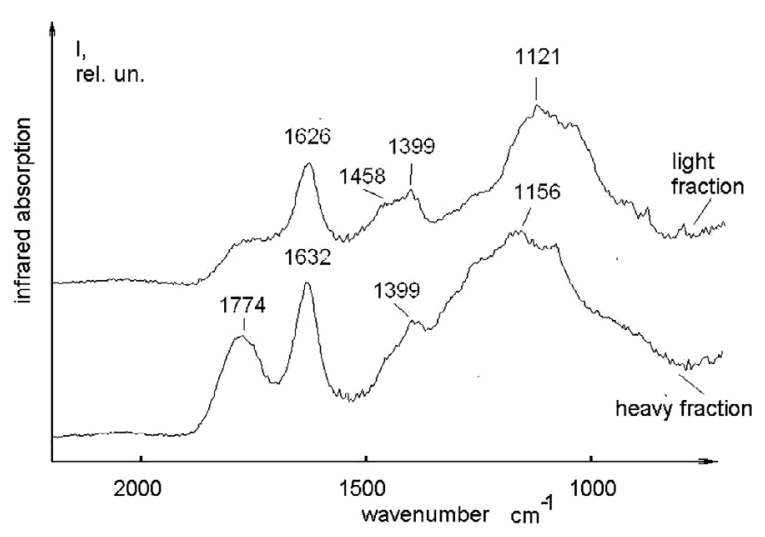

Fig. 3. Infrared spectra of the light and heavy fractions (3000 rpm centrifugation) for DCM-2-1, which was synthesized under molecular nitrogen TNB/RDX and isolated by hydrochloric acid oxidation.

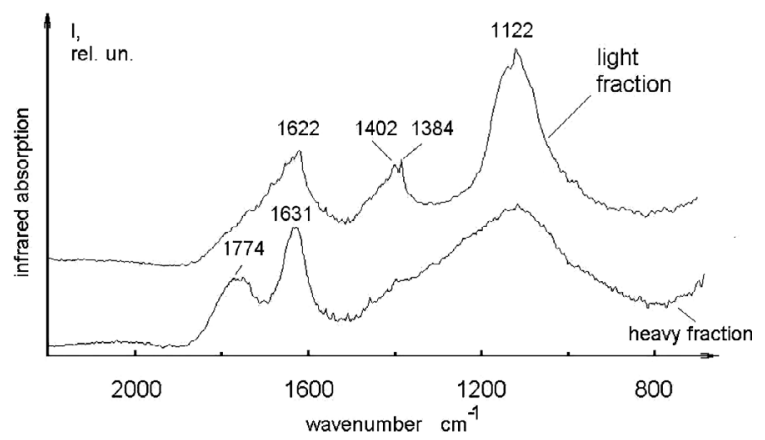

Fig. 4. Infrared spectra of the light and heavy fractions (3000 rpm centrifugation) for DCM-2-2, which was synthesized under molecular nitrogen and isolated by hydrochloric acid oxidation.

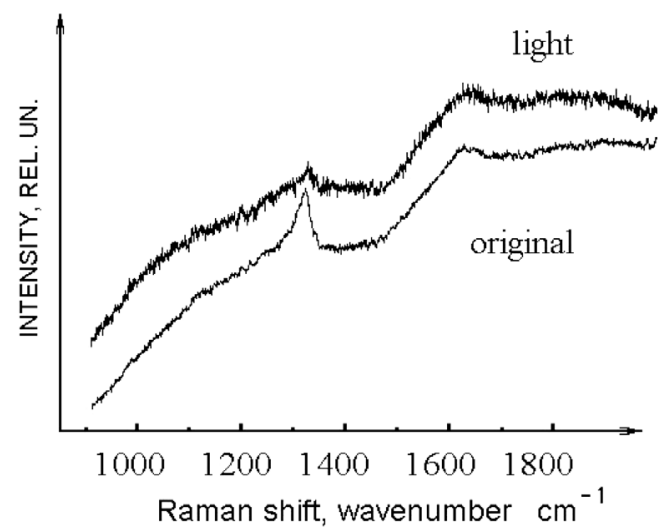

Fig. 5. Raman spectra of the light fraction (3000 rpm centrifugation) and the original sample DCM-2-2 (TNR/RDX or picric acid/RDX high explosives).

12-25 $2 \Theta$ region was considerable and higher than that of the diamond band (Fig. 8).

Raman spectra of the light fractions for DCM-1 and DCM-2 were also obtained (Figs. 4, 5, 7). In the Raman spectrum of the original sample, the diamond band is clearly seen. For the light fractions, this band is either absent [9] (for samples after $7000 \mathrm{rpm}$ centrifugation) or has low intensity. It follows that, for this type of the small

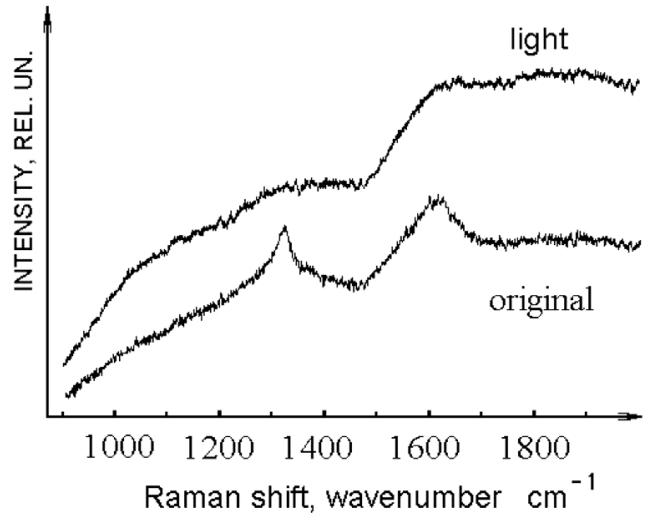

Fig. 6. Raman spectra of the light fraction (3000 rpm centrifugation) and the original sample DCM-2-1 (TNB/RDX).

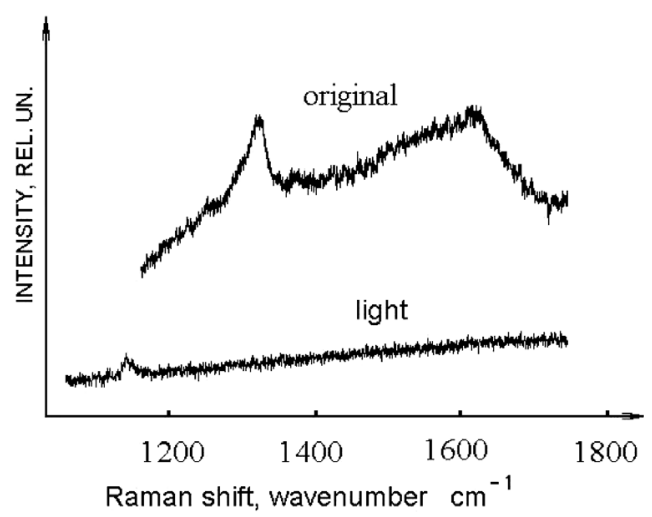

Fig. 7. Raman spectra of the original sample DCM-1 and the light fraction after $7000 \mathrm{rpm}$ centrifugation.
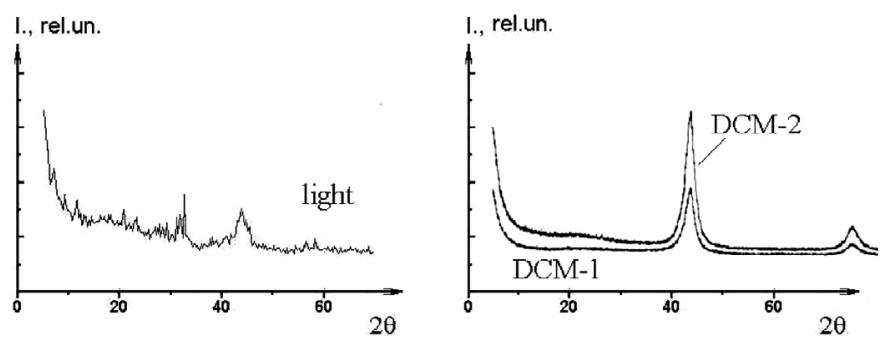

Fig. 8. X-ray diffraction patterns for the light fraction separated by $3000 \mathrm{rpm}$ centrifugation of DCM- 1 .

particles, a transformation from delocalized electron density to covalent bonding has likely taken place.

It is likely that DCM is an independent heterogeneous dense phase which is characterized by the variable ratio of the diamond $\left(s p^{3}\right)$ and non-diamond components, which include $s p^{2}$-carbon, molecular fragments and functional groups. The variation of the diamond and non-diamond components in a given sample makes this product independent and leads to scatter of the main material characteristics, which were discussed in [8]. Possibly due to the high density and the covalent bonding of DCM, the chemical stability of this material is relatively high. The distribution of $s p^{3}$-grains in the particles is of complicated 
character. In the fine particles, carbon $s p^{3}$-grains are either absent [9] or their concentration is small.

Note also that in a previous work [8], the authors tried to divide the surface groups from other admixtures in DCM to explain the similarity of Raman and IR-spectra (500-650 cm ${ }^{-1}, 1240-1320 \mathrm{~cm}^{-1}, 1590-1640 \mathrm{~cm}^{-1}, 3100$ $3500 \mathrm{~cm}^{-1}$ ) from the standpoint of the structural heterogeneity influence.

\section{Discussion}

The variable ratio of the diamond and non-diamond components in DCM as revealed by IR and Raman spectra means that the particles of this material are characterized by gradations in density or contrast. This implies that the formation of this material took place under nonequilibrium detonation conditions with considerable variation of pressure or substance density. An increasing entropy is recognized as the main non-equilibrium tendency during detonation. It is logical to assume that the intermediate species in DCM synthesis are radicals or other fragments of molecules formed during detonation. Any molecule or a molecular fragment can be characterized by the energies of its rotational, vibrational, and electronic states. From this standpoint, the increase in entropy reflects an increase in the number of states (possibilities) of these molecular fragments. In a non-equilibrium system, the developing processes lead to an increase in entropy or to a maximum disorder. Non-equilibrium energy can cause qualitative and quantitative changes at the molecular level. In the following discussion, we will omit discussion of the rotational energy level. We first consider the vibrational energy. The number of degrees of freedom of a molecule or fragment increase with the number of atoms in the molecule. In a high explosive mixture, incomplete destruction of molecules and further reaction of these products can give species with increased number of atoms and consequently with a large number of vibrational states. Higher dimensional structures, such as three-dimensional clusters instead of one-dimensional structures, also have greater degrees of freedom. In the systems studied here, disordered three-dimensional structures (or clusters) should generate the maximum disorder. Based on the inhomogeneity observed in the products, we expect that the probability of formation of threedimensional structures is higher compared to two- or onedimensional structures. Their stability is not critical for a non-equilibrium process.

It should also be noted that in terms of nonequilibrium processes (producing the maximum variety in the product), the probability of one- and two-dimensional structures should be nonzero. For the non-equilibrium system, it does not matter if these structures are carbon or organic fragments. The presence of one- and twodimensional structures is another reason for structural heterogeneity that develops when DCM are synthesized under non-equilibrium conditions. If carbon content is high, the three-dimensional or tetrahedral hybridization
Table 2. Dependence of the DCM output on the original high explosive (HE) density [12]. CC is condensed carbon.

\begin{tabular}{cccc}
\hline Mass of HE, g & $\begin{array}{r}\text { Density of } \\
\mathrm{HE}, \mathrm{g} / \mathrm{cm}^{3}\end{array}$ & $\begin{array}{c}\text { Mass of CC/ } \\
\text { Mass of HE, \% }\end{array}$ & $\begin{array}{c}\text { Mass of DCM/ } \\
\text { Mass of CC, \% }\end{array}$ \\
\hline 82.05 & 1.370 & 7.86 & 17.6 \\
81.40 & 1.372 & 8.19 & 15.6 \\
70.62 & 1.450 & 9.14 & 25.0 \\
71.31 & 1.480 & 8.70 & 36.8 \\
76.72 & 1.560 & 9.40 & 51.2 \\
77.20 & 1.580 & 9.60 & 58.0 \\
80.31 & 1.640 & 9.70 & 57.0 \\
\hline
\end{tabular}

of electron orbitals under equilibrium conditions are likely to transform into diamond-like carbon or diamond grains. The two-dimensional structures are typical of trigonalhybridized or graphite-like carbon.

Thus at this energetic level, the non-equilibrium process manifests through the facile formation of unstable volume structures. It can cause formation of $s p^{3}$ carbon areas in condensation products with a high carbon concentration.

We next discuss the electronic states. As noted, primary molecules under the detonation conditions are largely decomposed. Apparently, the non-equilibrium tendency (toward maximum disorder) will give rise to destruction products with different impulses resulting in a variety of energies in the products. This in turn gives a non-equilibrium density distribution or density fluctuations.

The experiments showed that the initial high explosive density (and consequently the substance density under the explosion condition) influenced on the DCM output (Tab. 2) and temperature of the synthesis determined the diamond grain size. Most likely the output of DCM is limited by the impossibility of producing denser explosives (Tab. 2) [2,12,13]. As a first approximation effectiveness of the synthesis can be presented by some function $F=\left(a \rho^{b} k T\right) / V$, where $b$ and $a$ are parameters, $\rho-$ is the substance density under explosion conditions, $k$ is the Boltzmann constant, and $V$ is the primary structure volume. This function involves some information on the density fluctuation. On the other hand, according to [12], a constant output of DCM ( 5 wt.\% of condensed carbon) was already observed under 8-10 GPa pressure, i.e. in the area of graphite stability. It is unlikely that the equilibrium thermodynamics is responsible for this experimental behavior [2,12].

Thus non-equilibrium conditions tend to transform the high energy, partially or completely, into density variations in the products. Large variations or fluctuations in density during synthesis are believed to be the reason for the appearance of compression and rarefaction dynamic areas or centers. The compression areas squeeze the carbon volume to a considerable density. This will result in 
the formation of diamond-like structures under interaction with the preservation atmosphere. The density variation is then reflected in the structure of the DCM particles. Note that the density of diamond-like carbon is possibly as high as $3.3 \mathrm{~g} / \mathrm{cm}^{3}$. It is likely that the molecular fragments represent $0.5-1.5 \mathrm{~g} / \mathrm{cm}^{3}$. The average density in the reaction zone of a detonation wave for these high explosives is close to $2.0 \mathrm{~g} / \mathrm{cm}^{3}$, which is practically in the middle of these values.

To relate these ideas to the detonation process, smallangle X-Ray scattering (SAXS) using synchrotron radiation in the explosion products should be used to probe the explosion products. SAXS has been reported to measure density contrast or density fluctuations [14-17], although this method does not identify what mechanisms lead to the observed density variations [17]. The question arises as to what extent the density can fluctuate under nonequilibrium explosion conditions [14].

Thus it is most likely that the density fluctuations of a few to tens nanometers occurs under non-equilibrium DCM synthesis.

\section{Conclusions}

Raman, XRD, and IR spectra of diamond-containing material and of the separated DCM fractions were obtained. The particles of this heterogeneous material were characterized by the variable ratio of the diamond $\left(s p^{3}\right)$ and non-diamond components as well as by their density variation. The fine DCM particles contained an insignificant amount of $s p^{3}$ carbon. We suggest that a non-equilibrium detonation synthesis producing density fluctuations was the main reason for this heterogeneity.

The authors acknowledge the Department of Chemistry (SFU).

\section{References}

1. R.N. Greiner, D.S. Phillips, J.D. Johnson, F. Volk, Nature 333, 440 (1988)

2. V.M. Titov, V.F. Anisichkin, I.Yu. Mal'kov, Combust. Explos. Shock Waves 25, 117 (1989)

3. A. Krueger, M. Ozawa, G. Jarre, Y. Liang, J. Stegk, L. Li, Phys. Stat. Sol. 204, 2881 (2007)

4. I.S. Larionova, A.V. Frolov, L.I. Poleva, N.V. Bychin, Colloid J. 66, 372 (2004)

5. V. Mochalin, S. Osswald, Y. Gogotsi, Chem. Mater. 21, $273(2009)$

6. B.V. Spitsyn, J.L. Davidson, M.N. Gradoboev et al., Diam. Relat. Mater. 15, 296 (2006)

7. V. Pichot, M. Comet, C. Baras, E. Fousson, A. Senger, F. Le Normand, D. Spitzer, Diam. Relat. Mater. 17, 13 (2008)

8. A.Ya. Korets, A.S. Krylov, E.V. Mironov, Russ. J. Phys. Chem. B 2, 485 (2007)

9. A.Ya. Korets, A.S. Krylov, E.V. Mironov, in Proc. 25th Int. Conf. on Equations of State for Matter Physics of Extreme States of Matter, Elbrus, Russia, 2010, p. 51

10. E. Mironov, A. Koretz, E. Petrov, Diam. Relat. Mater. 11, $872(2002)$

11. A.Ya. Korets, E.V. Mironov, E.A. Petrov, Combust. Explos. Shock Waves 39, 464 (2003)

12. I.Yu. Malkov, in Proc. Conf. Ultradisperse powders, materials, and nanostructures, Krasnoyarsk, Russia, 1996, p. 17 (in Russian)

13. I.Yu. Malkov, L.I. Filatov, V.M. Titov et al., Combust. Explos. Shock Waves 29, 131 (1993)

14. A.L. Kupershtokh, A.P. Ershov, D.A. Medvedev, Combust. Explos. Shock Waves 34, 460 (1998)

15. A.N. Aleshaev, P.I. Zubkov, G.N. Kulipanov et al., Combust. Explos. Shock Waves 37, 585 (2001)

16. V.M. Titov, B.P. Tolochko, K.A. Ten, L.A. Lukyanchikov, E.R. Pruuel, Diam. Relat. Mater. 16, 2009 (2007)

17. A.P. Ershov, Tech. Phys. Lett. 27, 841 (2001) 